\title{
Predictors of Stroke Outcome Extracted from Multivariate Linear Discriminant Analysis or Neural Network Analysis
}

\author{
Tomohisa Nezu ${ }^{1}$, Naohisa Hosomi ${ }^{2,}{ }^{3}$, Kazumasa Yoshimura4 ${ }^{4}$, Daisuke Kuzume ${ }^{2}$, Hiroyuki Naito ${ }^{1}$, \\ Shiro Aoki ${ }^{1}$, Yuko Morimoto ${ }^{2}$, Masato Kinboshi ${ }^{2}$, Takeshi Yoshida ${ }^{5}$, Yuji Shiga ${ }^{1}$, Naoto Kinoshita ${ }^{1}$, \\ Akira Furui ${ }^{4}$, Genta Tabuchi ${ }^{4}$, Hiroki Ueno ${ }^{1}$, Toshio Tsuji ${ }^{4}$ and Hirofumi Maruyama ${ }^{1}$
}

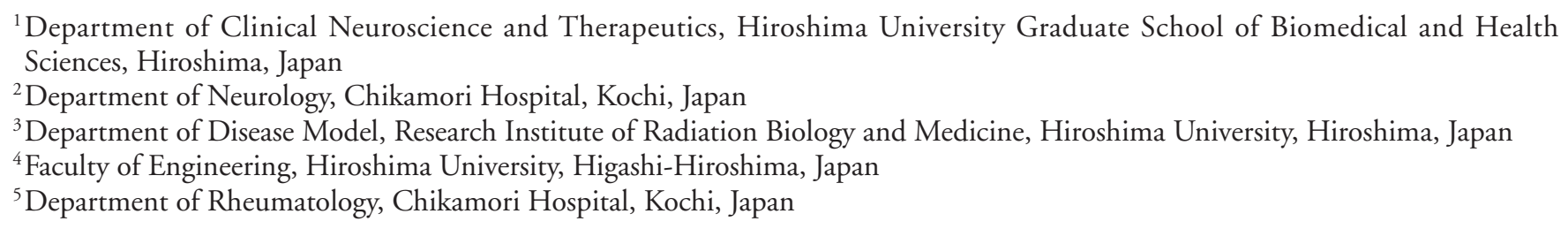

Aim: The prediction of functional outcome is essential in the management of acute ischemic stroke patients. We aimed to explore the various prognostic factors with multivariate linear discriminant analysis or neural network analysis and evaluate the associations between candidate factors, baseline characteristics, and outcome.

Methods: Acute ischemic stroke patients $(n=1,916)$ with premorbid modified Rankin Scale $(\mathrm{mRS})$ scores of 0-2 were analyzed. The prediction models with multivariate linear discriminant analysis (quantification theory type II) and neural network analysis (log-linearized Gaussian mixture network) were used to predict poor functional outcome (mRS 3-6 at 3 months) with various prognostic factors added to age, sex, and initial neurological severity at admission.

Results: Both models revealed that several nutritional statuses and serum alkaline phosphatase (ALP) levels at admission improved the predictive ability. Of the 1,484 patients without missing data, 560 patients $(37.7 \%)$ had poor outcomes. The patients with poor outcomes had higher ALP levels than those without $(294.3 \pm 259.5$ vs. $246.3 \pm 92.5 \mathrm{U} / 1, P<0.001)$. Multivariable logistic analyses revealed that higher ALP levels (1-SD increase) were independently associated with poor stroke outcomes after adjusting for several confounding factors, including the neurological severity, malnutrition status, and inflammation (odds ratio 1.21, 95\% confidence interval $1.02-$ 1.49). Several nutritional indicators extracted from prediction models were also associated with poor outcome.

Conclusion: Both the multivariate linear discriminant and neural network analyses identified the same indicators, such as nutritional status and serum ALP levels. These indicators were independently associated with functional stroke outcome.

Key words: Neural network analysis, Acute ischemic stroke, Outcome

\section{Introduction}

Predicting functional outcomes after acute ischemic stroke is important because patients with physical disability at hospital discharge require varying levels of rehabilitation or long-term care after discharge. Neurological severity at stroke onset is one of the most important factors for predicting long-term functional outcomes. Simple and reliable assessments based on age and the National Institutes of Health Stroke Scale (NIHSS) score have been widely used to predict functional outcomes among acute stroke

Address for correspondence: Tomohisa Nezu, Department of Clinical Neuroscience and Therapeutics, Hiroshima University Graduate School of Biomedical Sciences, 1-2-3 Kasumi, Minami-ku, Hiroshima, Hiroshima 734-8551, Japan E-mail: tomonezu@hiroshima-u.ac.jp

Received: August 3, 2020 Accepted for publication: October 12, 2020

Copyright@2021 Japan Atherosclerosis Society

This article is distributed under the terms of the latest version of CC BY-NC-SA defined by the Creative Commons Attribution License. 
patients $^{1-3)}$. In clinical settings, blood laboratory and neurological findings are essential for acute ischemic stroke. We reported previously that malnutrition status based on laboratory findings at admission is independently associated with poor stroke outcome after adjusting for baseline characteristics, including age, sex, and the NIHSS score ${ }^{4)}$. In that study, nutritional status was evaluated using the Controlling Nutritional Status (CONUT) score ${ }^{5)}$, which is calculated from the serum albumin concentration, total peripheral lymphocyte count, and total cholesterol level. Conversely, several other simple scores calculated from laboratory findings, including lymphocyte count, neutrophil count, platelet count, albumin levels, and C-reactive protein levels, were proposed. These indicators are also associated with stroke outcomes ${ }^{6-10)}$. Other studies have shown that hemoglobin levels, plasma D-dimer levels, and decreased estimated glomerular filtration rate are associated with stroke outcomes ${ }^{11-14)}$. Although accumulated evidence suggested that blood laboratory findings at admission are useful for predicting stroke outcomes, weighing those laboratory parameters relative to each other or evaluating their priority because of the various fluctuations in the ranges of laboratory findings are sometimes difficult. Additionally, it remains unclear which laboratory parameters at admission, besides age, sex, and the NIHSS score, improve the predictive ability for stroke outcomes.

Machine learning has recently been applied to predict clinical outcomes in several medical fields. This method may provide a promising factor or scoring system for predicting clinical outcomes unlike traditional analysis models based on the assumption of a linear relationship between selected variables and the $\log$ odds of outcomes. The log-linearized Gaussian mixture network (LLGMN), a kind of machine learning method, has been used for pattern classification problems of various bioelectric signals ${ }^{15-17)}$. In the present study, we aimed to find useful blood laboratory parameters besides age, sex, and neurological severity (the NIHSS score) to improve the predictive ability for acute ischemic stroke outcome using both traditional and neural network analyses. Additionally, we evaluated the clinical significance of indicators extracted from those analyses to correspond to the stroke functional outcome.

\section{Methods}

\section{Study Population}

This was a double-center, hospital-based retrospective study involving patients with acute ischemic stroke within 7 days after stroke onset hospitalized at Hiroshima University and Chikamori Hospital between October 2009 and September 2018. Patients with intravenous thrombolysis or endovascular treatment were excluded from this study. A total of 2,359 consecutive patients were enrolled. This study complies with the Declaration of Helsinki guidelines for investigations involving humans, and the study protocol was approved by the Ethics Committees of Hiroshima University and Chikamori Hospital. This study was conducted under the opt-out method, as it was conducted retrospectively using clinical records.

\section{Assessment of Clinical Characteristics}

The following clinical characteristics were recorded at admission: age, sex, body mass index (BMI), and classical vascular risk factors including hypertension, diabetes mellitus, dyslipidemia, chronic kidney disease (CKD), atrial fibrillation, daily alcohol intake $(>40 \mathrm{~g})$, smoking habit (current smokers or non-current smokers), and history of stroke and ischemic heart disease. Criteria for hypertension, diabetes mellitus, dyslipidemia, and atrial fibrillation were previously defined ${ }^{4)}$. CKD was evaluated by eGFR using the equation of the Japanese Society of Nephrology: eGFR $=194 \times$ creatine $(\mathrm{Cr})^{-1.094} \times$ age $^{-0.287}$ $\left(\mathrm{mL} / \mathrm{min} / 1.73 \mathrm{~m}^{2}\right)$. For women, the eGFR was multiplied by a correction factor of $0.739^{18)}$. CKD was defined as an eGFR level $<60 \mathrm{~mL} / \mathrm{min}$ per 1.73 $\mathrm{m}^{2}$. Atrial fibrillation was diagnosed when a previous electrocardiography (ECG) or ECG conducted on admission revealed atrial fibrillation. Neurological severity was assessed according to the NIHSS score. Stroke subtypes were classified according to the criteria established by the Trial of ORG 10172 in Acute Stroke Treatment classification ${ }^{19)}$.

\section{Laboratory Parameters}

Supplemental Table 1 lists the laboratory parameters. These parameters are basically used to manage patients with acute stroke in daily clinical settings in our hospitals. Most blood samples were collected at admission, and some findings, such as hemoglobin A1c (HbA1c) or cholesterol levels, were obtained within 2 days after admission. We calculated the following scores reflected as nutritional status or inflammation. The CONUT scores were calculated from the serum albumin concentration, total peripheral lymphocyte count, and total cholesterol concentration ${ }^{5)}$. The Geriatric Nutritional Risk Index (GNRI) was calculated from serum albumin levels and $\mathrm{BMI}^{20)}$. The NLR (neutrophil-to-lymphocyte ratio), PLR (platelet-to-lymphocyte ratio), and CAR (CRP- 
to-albumin ratio) were also calculated ${ }^{6,21)}$.

\section{Assessment of Stroke Outcomes}

The primary outcome was evaluated from the 3-month functional status. A poor outcome was defined as a score of 3-6 on the modified Rankin Scale (mRS); a score of 0-2 was defined as a good outcome. Secondary outcomes were an mRS score of $0-1$ at 3 months and death at 3 months. Essentially, attending physicians evaluated the mRS score at 3 months after stroke onset by examining the patients. When physicians were unable to examine the patients, the mRS score was assessed by the attending physicians based on a review of the medical records or contacting the caregivers of patients.

\section{Statistical Analysis}

Categorical variables are presented as numbers and percentages, and continuous variables are presented as the means with standard deviations (SDs) or medians (interquartile ranges). The statistical significance of intergroup differences was evaluated using the $\chi^{2}$ test for categorical variables and Student's $t$ test, Mann-Whitney $U$ test, or one-way analysis of variance for continuous variables as appropriate. The various baseline indicators listed in Supplemental Table 1 were added to the following prediction models. Quantification theory type II was used as a multivariate linear discriminant model based on the assumption of a linear relationship between selected variables and the $\log$ odds of outcomes ${ }^{22)}$. This method is a discriminant analysis of qualitative data with external criteria of nominal scale, and both dependent variables and covariates are treated as categorical data. This method is equivalent to a linear discriminant analysis using dummy variables. The LLGMN analysis was used as a neural network analysis for a multivariate nonlinear discriminant model. This network analysis allows us to estimate the statistical distribution of sample data based on machine learning and predict the posterior probability of the class for unknown input data. A detailed description of the methods used for the LLGMN analysis is provided in the Supplemental Materials. From the results of those prediction models, we focused on the ALP levels at admission. Spearman's correlation analysis was conducted to determine the associations between ALP levels and other laboratory findings. Receiver operating characteristic (ROC) curves were constructed to obtain the optimal cutoff ALP levels to predict poor stroke outcome (mRS score of 3-6 at 3 months). Multivariable logistic analysis was conducted to identify predictors for the primary outcome by a backward selection procedure using $P>$
0.10 of the likelihood ratio test as the exclusion criterion. Those analyses were conducted using JMP 14.0 (SAS Institute, Inc., Cary, NC, USA).

\section{Results}

Baseline Characteristics and Laboratory Findings to Improve the Predictive Ability for Stroke Outcome

A total of 2,359 consecutive patients were enrolled. The flow chart of patient selection is shown in Fig. 1A. Among these patients, 443 patients were excluded due to premorbid $\mathrm{mRS} \geq 3$. Of the remaining 1,956 patients, 737 patients were excluded due to lack of any abovementioned blood laboratory findings or lack of data on functional outcomes. Of the remaining 1,219 patients, 791 patients had good outcomes, and 428 patients had poor outcomes. Therefore, 800 patients were selected equally for good outcome $(n=400)$ or poor outcome $(n=400)$ by randomness extraction algorithms and analyzed using multivariate linear discriminant analysis (quantification theory type II) or LLGMN with 10 -fold cross-validation. The area under the curve (AUC) of variables including age, sex, and the NIHSS score (reference) to predict poor outcome was 0.776 using multivariate linear discriminant analysis. The AUC of those variables was 0.819 using LLGMN. After adding the various indicators to age, sex, and the NIHSS score, each AUC value was evaluated. Fig. 2 shows the indicators that ranked in the top 10 for improving the predictive ability. Both analyses revealed that several factors, such as ALP, CONUT score, albumin, and GNRI, were important factors for improving the predictive ability of AUC for poor stroke outcome.

The Associations between Alkaline Phosphatase and Baseline Characteristics

From the above results, we focused on the association between ALP values, baseline characteristics, and stroke outcomes. Fig. 1B shows the flow chart of patient selection. Finally, 1,484 patients were evaluated to analyze the associations between ALP, baseline characteristics, and stroke outcome. Table 1 shows the baseline characteristics of the patients who were divided into four groups according to the quartiles of ALP values $(\leq 192,193$ 238, 239-296, and $\geq 297 \mathrm{U} / \mathrm{l}$ ). Increased ALP values were associated with older age and higher prevalence of previous stroke $(P=0.016$ and 0.025$)$. Additionally, increased ALP values were associated with a higher GNRI and higher CAR $(P=0.030$ and $<0.001)$. Supplemental Table 2 shows Spearman's correlation 
Table 1. Baseline characteristics by quartiles of ALP values

\begin{tabular}{|c|c|c|c|c|c|}
\hline \multirow[b]{2}{*}{ Factors } & \multicolumn{5}{|c|}{ ALP Quartiles, U/l } \\
\hline & $\begin{array}{c}\mathrm{Q} 1(\leq 192) \\
(n=374)\end{array}$ & $\begin{array}{c}\text { Q2 }(193-238) \\
\quad(n=371)\end{array}$ & $\begin{array}{c}\text { Q3 (239-296) } \\
\quad(n=368)\end{array}$ & $\begin{array}{c}\mathrm{Q} 4(\geq 297) \\
(n=371)\end{array}$ & $p$ \\
\hline Age, years & $71.8 \pm 12.8$ & $73.0 \pm 11.6$ & $74.1 \pm 10.8$ & $74.1 \pm 10.5$ & 0.016 \\
\hline Sex, male & $238(63.6)$ & $238(64.2)$ & $227(61.7)$ & $221(59.6)$ & 0.56 \\
\hline BMI, $\mathrm{kg} / \mathrm{m}^{2}(n=1,463)$ & $23.4 \pm 3.6$ & $23.5 \pm 3.9$ & $23.2 \pm 3.9$ & $22.9 \pm 3.8$ & 0.19 \\
\hline Daily alcohol intake $(n=1,459)$ & $113(30.7)$ & $118(32.4)$ & $112(31.0)$ & $91(24.9)$ & 0.11 \\
\hline Current smoking $(n=1,460)$ & $67(18.2)$ & $89(24.4)$ & $84(23.3)$ & $73(20.0)$ & 0.14 \\
\hline Hypertension & $266(71.1)$ & $242(65.2)$ & $267(72.6)$ & $254(68.5)$ & 0.14 \\
\hline Diabetes mellitus $(n=1,483)$ & $134(35.9)$ & $136(36.7)$ & $113(30.7)$ & $131(35.3)$ & 0.32 \\
\hline Dyslipidaemia $(n=1,481)$ & $196(52.6)$ & $179(48.4)$ & $193(52.5)$ & $166(44.9)$ & 0.11 \\
\hline Chronic kidney disease & $150(40.1)$ & $156(42.1)$ & $147(40.0)$ & $136(36.7)$ & 0.51 \\
\hline Atrial fibrillation $(n=1,483)$ & $87(23.3)$ & $72(19.5)$ & $80(21.7)$ & $76(20.5)$ & 0.62 \\
\hline Previous stroke $(n=1,483)$ & $90(24.1)$ & $88(23.7)$ & $115(31.3)$ & $114(30.7)$ & 0.025 \\
\hline Previous ischaemic heart disease & $56(15.0)$ & $49(13.2)$ & $48(13.0)$ & $51(13.7)$ & 0.87 \\
\hline NIHSS score at admission & $3(1-6)$ & $3(1-5)$ & $3(1-7.5)$ & $3(1-7)$ & 0.46 \\
\hline Stroke subtype & & & & & 0.31 \\
\hline Small-vessel occlusion & $90(24.1)$ & $99(26.7)$ & $98(26.6)$ & $103(27.8)$ & \\
\hline Large-artery atherosclerosis & $79(21.1)$ & $95(25.6)$ & $89(24.2)$ & $92(24.8)$ & \\
\hline Cardioembolic stroke & $111(29.7)$ & $86(23.2)$ & $107(29.1)$ & $87(23.5)$ & \\
\hline Other aetiology & $94(25.1)$ & $91(24.5)$ & $74(20.1)$ & $89(24.0)$ & \\
\hline \multicolumn{6}{|l|}{ Laboratory scores } \\
\hline CONUT $(n=1,477)$ & $1(0.5-3)$ & $1(0-2)$ & $1(0-3)$ & $2(0-3)$ & 0.07 \\
\hline GNRI $(n=1,454)$ & $105.1(98.4-111.9)$ & $105.5(98.4-111.5)$ & $104.8(97.8-111.1)$ & $103.2(94.4-110.6)$ & 0.030 \\
\hline $\operatorname{NLR}(n=1,479)$ & $2.8(1.8-4.7)$ & $2.9(2.0-4.1)$ & $3.0(1.9-5.3)$ & $3.0(2.0-5.0)$ & 0.37 \\
\hline $\operatorname{PLR}(n=1,479)$ & $124.7(95.3-171.2)$ & $124.4(98.4-166.0)$ & $131.1(97.8-184.4)$ & $133.0(92.4-184.4)$ & 0.39 \\
\hline CAR $(n=1,470)$ & $0.03(0.02-0.09)$ & $0.05(0.02-0.10)$ & $0.05(0.02-0.13)$ & $0.06(0.02-0.23)$ & $<0.001$ \\
\hline
\end{tabular}

Data are presented as the means \pm standard deviation for age, BMI, and each laboratory finding; as median (interquartile range) for NIHSS score at admission, CONUT, GNRI, NLR, PLR, and CAR; and as number of patients (\%) for others.

ALP, alkaline phosphatase; BMI, body mass index; NIHSS, National Institutes Health Stroke Scale. CONUT, Controlling Nutritional Status; GNRI, Geriatric Nutritional Risk Index; NLR, neutrophil-to-lymphocyte ratio; PLR, platelet-to-lymphocyte ratio; CAR, C reactive protein-toalbumin ratio

analysis between ALP values and other laboratory findings. Lower HDL, higher AST, higher ALT, higher LDH, higher $y$-GTP, higher CRP, and higher D-dimer levels were closely associated with higher ALP levels $(P<0.001$, respectively).

\section{The Associations between Alkaline Phosphatase and Stroke Outcomes}

Of 1,484 patients, 560 patients $(37.7 \%)$ had poor outcomes (mRS score of 3-6) at 3 months after stroke onset. The patients with poor stroke outcomes were older and less likely to be male; had lower BMI, frequency of daily alcohol intake, and current smoking; had a higher frequency of CKD, atrial fibrillation, and previous stroke; and exhibited more severe NIHSS scores than those with good outcomes (Table 2). The patients with poor outcomes had higher ALP levels than those with good outcomes
$(294.3 \pm 259.5$ vs. $246.3 \pm 92.5 \mathrm{U} / 1, \quad P<0.001)$. Similarly, the patients with poor outcomes had higher CONUT, GNRI, NLR, PLR, and CAR than those with good outcomes $(P<0.001)$. Supplemental Table 3 shows other blood laboratory findings between the two groups. The optimal cutoff ALP level to predict patients with poor outcomes was $\geq 288 \mathrm{U} / \mathrm{l}$, with a sensitivity of $55 \%$, a specificity of $59 \%$, and an area under the ROC curve of 0.578. Multivariable logistic analyses were conducted using the following three models. The variables included in model 1 were age, sex, BMI, daily alcohol intake, current smoking, comorbidities (hypertension, diabetes mellitus, dyslipidemia, CKD, and atrial fibrillation), previous stroke, previous ischemic heart disease, the NIHSS score, and ALP levels (quartiles range, 1-SD increase or optimal cutoff ALP level $\geq 288 \mathrm{U} / \mathrm{l}$ ). For model 2, the other blood laboratory findings were added to 

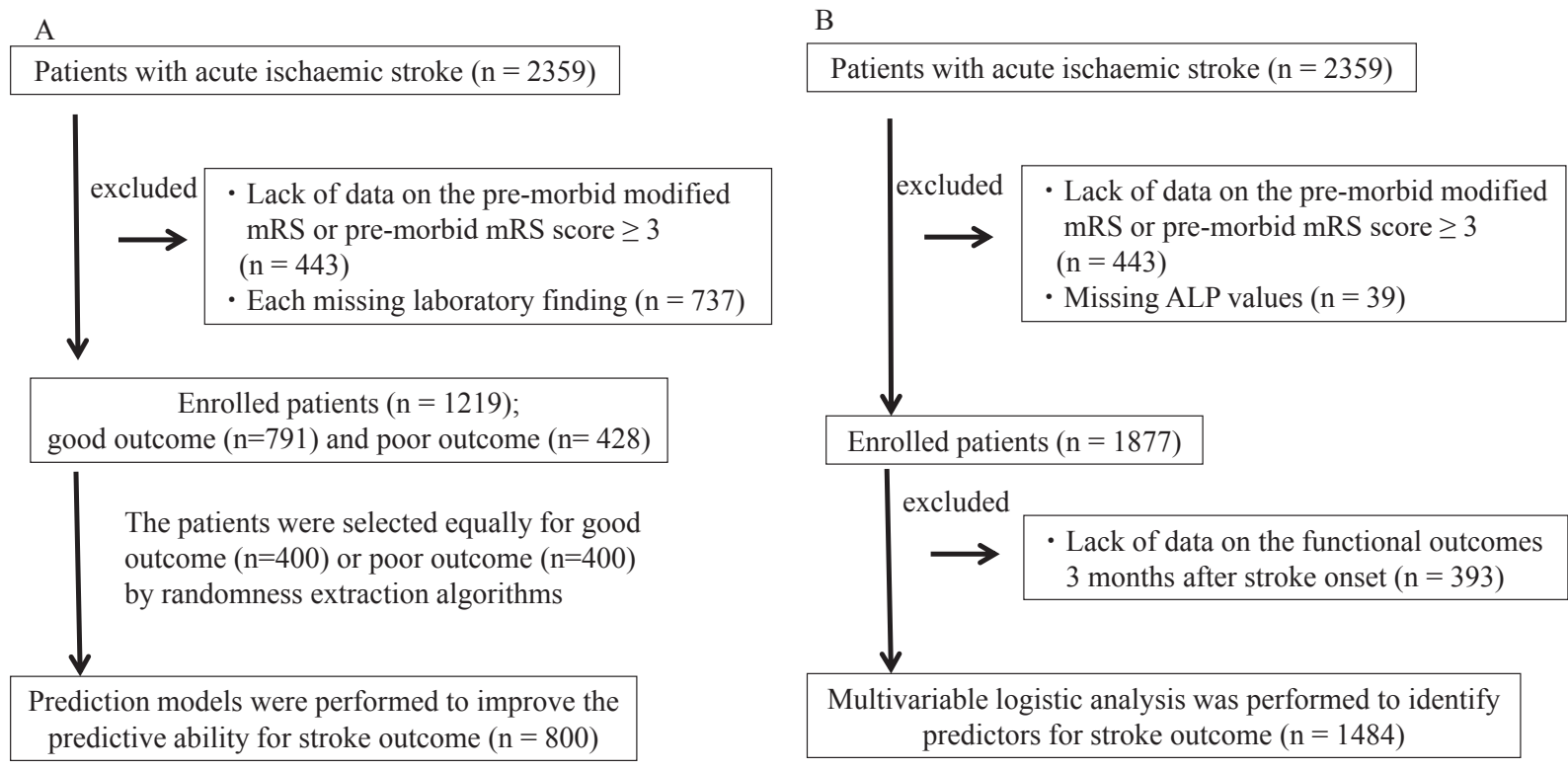

Fig. 1.

Flowchart of patient selection for (A) the prediction models for multivariate linear discriminant analysis (quantification theory type II) and neural network analysis (log-linearized Gaussian mixture network) and (B) the association between alkaline phosphatase (ALP) levels, baseline characteristics, and stroke outcome.

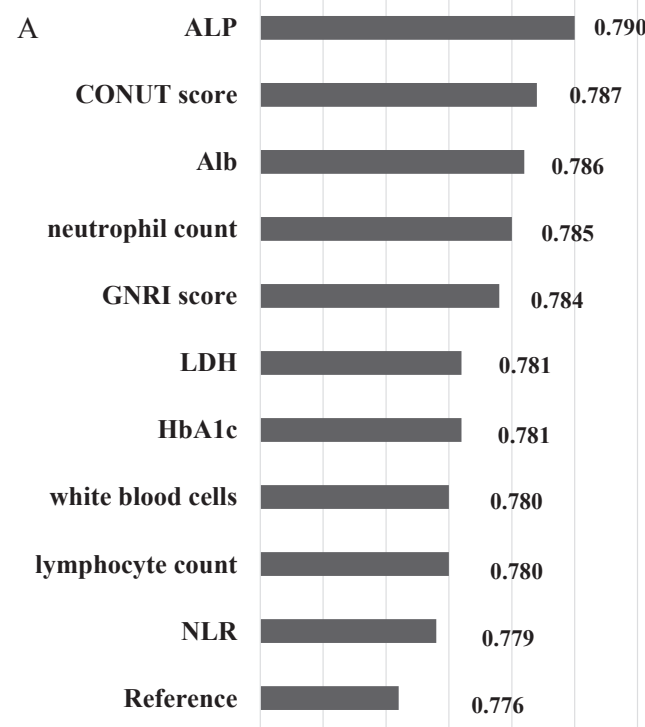

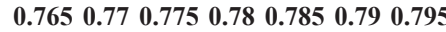
AUC values

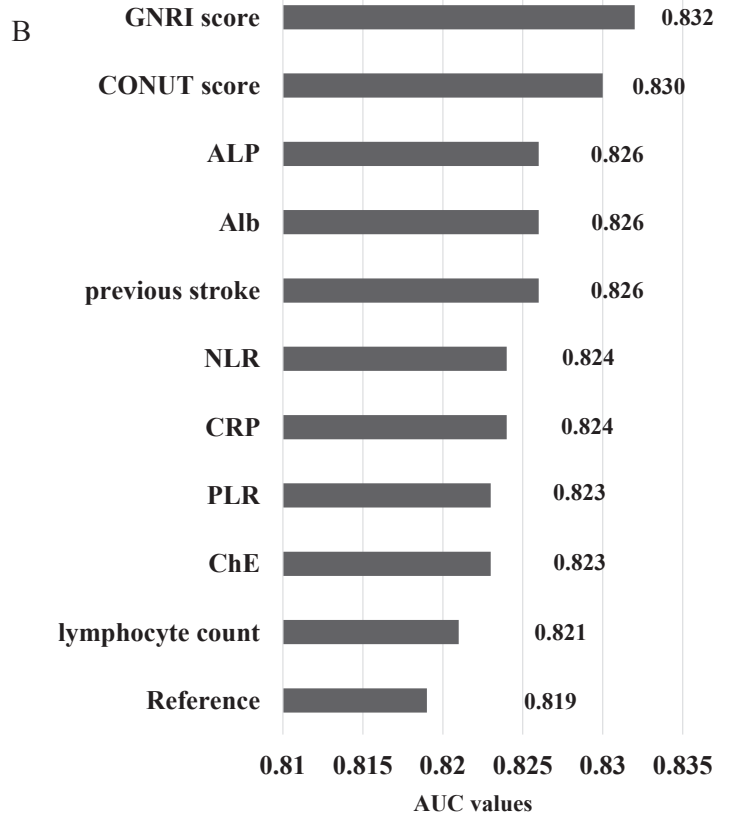

AUC values

Fig. 2. The area under the curve (AUC) from the receiver operating characteristic curve was used to predict stroke outcome of the 10 highest ranked variables

(A) Multivariate linear discriminant analysis, (B) log-linearized Gaussian mixture network analysis. References indicated age, sex, and National Institutes of Health Stroke Scale score.

ALP, alkaline phosphatase; CONUT, Controlling Nutritional Status; Alb, albumin, GNRI, Geriatric Nutritional Risk Index; LDH, lactate dehydrogenase, NLR, neutrophil-to-lymphocyte ratio; CRP, C-reactive protein; PLR, platelet-to-lymphocyte ratio; ChE, cholinesterase. 
Table 2. Baseline characteristics associated with 3-month functional outcome

\begin{tabular}{|c|c|c|c|}
\hline & $\operatorname{mRS} 0-2(n=960)$ & mRS 3-6 $(n=524)$ & $p$ \\
\hline Age, years & $70.8 \pm 11.5$ & $77.8 \pm 9.9$ & $<0.001$ \\
\hline Sex, male & $640(66.7)$ & $284(54.2)$ & $<0.001$ \\
\hline BMI, $\mathrm{kg} / \mathrm{m}^{2}(n=1,463)$ & $23.6 \pm 3.7$ & $22.6 \pm 3.8$ & $<0.001$ \\
\hline Daily alcohol intake $(n=1,459)$ & $310(32.7)$ & $124(24.3)$ & $<0.001$ \\
\hline Current smoking $(n=1,460)$ & $240(25.3)$ & $73(14.3)$ & $<0.001$ \\
\hline Hypertension & $658(68.5)$ & $371(70.8)$ & 0.38 \\
\hline Diabetes mellitus ( $n=1,483)$ & $336(35.0)$ & $178(34.0)$ & 0.73 \\
\hline Dyslipidaemia $(n=1,481)$ & $493(51.4)$ & $241(46.3)$ & 0.06 \\
\hline Chronic kidney disease & $340(35.4)$ & $249(47.5)$ & $<0.001$ \\
\hline Atrial fibrillation $(n=1,483)$ & $156(16.3)$ & $159(30.4)$ & $<0.001$ \\
\hline Previous stroke $(n=1,483)$ & $238(24.8)$ & $169(32.3)$ & 0.002 \\
\hline Previous ischaemic heart disease & $120(12.5)$ & $84(16.0)$ & 0.06 \\
\hline NIHSS score at admission & $2(1-4)$ & $7(3-17)$ & $<0.001$ \\
\hline Stroke subtype & & & $<0.001$ \\
\hline Small-vessel occlusion & $281(29.3)$ & $109(20.8)$ & \\
\hline Large-artery atherosclerosis & $242(25.2)$ & $113(21.6)$ & \\
\hline Cardioembolic stroke & $211(22.0)$ & $180(34.4)$ & \\
\hline Other aetiology & $226(23.5)$ & $122(23.3)$ & \\
\hline Laboratory findings & $71(29.3)$ & $49(35.0)$ & \\
\hline ALP levels U/l & $246.3 \pm 92.5$ & $294.3 \pm 259.5$ & $<0.001$ \\
\hline CONUT $(n=1,477)$ & $1(0-2)$ & $2(1-3)$ & $<0.001$ \\
\hline GNRI $(n=1,454)$ & $106.3(100.1-112.8)$ & $100.4(92.8-107.6)$ & $<0.001$ \\
\hline $\operatorname{NLR}(n=1,479)$ & $2.6(1.8-3.9)$ & $3.9(2.3-6.6)$ & $<0.001$ \\
\hline $\operatorname{PLR}(n=1,479)$ & $122.8(93.8-168.4)$ & $142.6(100.0-204.7)$ & $<0.001$ \\
\hline CAR $(n=1,470)$ & $0.04(0.02-0.09)$ & $0.07(0.03-0.34)$ & $<0.001$ \\
\hline
\end{tabular}

Data are presented as the means \pm standard deviation for age, BMI, and each laboratory finding; as median (interquartile range) for NIHSS score at admission, CONUT, GNRI, NLR, PLR, and CAR; and as number of patients (\%) for others. ALP, alkaline phosphatase; BMI, body mass index; NIHSS, National Institutes Health Stroke scale. CONUT, Controlling Nutritional Status; GNRI, Geriatric Nutritional Risk Index; NLR, neutrophil-to-lymphocyte ratio; PLR, platelet-tolymphocyte ratio; CAR, $\mathrm{C}$ reactive protein-to-albumin ratio

model 1. For model 3, scores calculated from blood laboratory findings (CONUT scores, GNRI, NLR, PLR, and CAR) were added to model 1. Multivariable analysis revealed that increased ALP levels were independently associated with poor stroke outcome after adjusting for several baseline characteristics and laboratory findings (Table 3). For other candidate parameters (albumin, CONUT score, and GNRI) extracted from prediction models, ALP levels as a 1-SD increase were adopted as follows. Serum albumin levels were independently associated with poor stroke outcome (odds ratio (OR) 0.46, 95\% confidence interval (CI) 0.34-0.61, $P<0.001$, model 2). Higher CONUT score and lower GNRI were also associated with poor stroke outcome (OR 1.15, 95\% CI $1.04-1.26, P=0.006$ and OR $0.98,95 \%$ CI $0.96-$ $0.99, P=0.008$, respectively, model 3 ).

Of 1,484 patients, 83 patients $(5.6 \%)$ had died at 3 months after stroke onset. The patients who had died by 3 months had higher ALP levels than survivors
(412.6 \pm 565.2 vs. $254.4 \pm 107.0 \mathrm{U} / 1, \quad P<0.001)$. Multivariable analysis revealed that a $1-S D$ increase in ALP levels was independently associated with mortality (OR 1.42, 95\% CI 1.18-1.70, $P<0.001$, model 1 , and OR 1.40, 95\% CI 1.11-1.76, $P<$ 0.001 , model 3) although these associations were not significant according to the analysis of model 2 (OR $1.30,95 \%$ CI $0.98-1.73, P=0.067$ ).

For the analysis of secondary outcome on mRS scores of $0-1$ at 3 months, 194 patients with a premorbid mRS score of 2 were excluded. Of the remaining 1,290 patients, 699 patients had mRS scores of $0-1$ at 3 months $(54.2 \%)$. The patients with $\mathrm{mRS}$ scores of 2-6 had higher ALP levels than those with $\mathrm{mRS}$ scores of $0-1(278.1 \pm 214.5$ vs. $245.7 \pm$ 93.7 U/l, $P=0.001)$. Multivariable analysis revealed that a 1-SD increase in ALP levels was independently associated with mRS scores of 2-6 at 3 months (OR $1.2195 \%$ CI $1.02-1.45, P=0.015$, model 1 , and OR 1.26 , 95\% CI 1.03-1.54, $P=0.014$, model 3), 
Table 3. Odds ratio (95\% CI) for poor functional outcome at 3 months by quartiles, 1-SD change and optimal cut-off value in alkaline phosphatase levels

\begin{tabular}{lcccccc}
\hline & \multicolumn{9}{c}{ ALP Quartiles, U/L } & 1-SD increase in & ALP $(\geq 288)$ vs. \\
\cline { 2 - 5 } & $\begin{array}{c}\text { Q1 }(\leq 192) \\
(n=374)\end{array}$ & $\begin{array}{c}\text { Q2 }(193-238) \\
(n=371)\end{array}$ & $\begin{array}{c}\text { Q3 }(239-296) \\
(n=368)\end{array}$ & $\begin{array}{c}\text { Q4 }(\geq 297) \\
(n=371)\end{array}$ & $\begin{array}{c}\text { ALP levels } \\
\text { ALP }(<288)\end{array}$ \\
\hline Model 1 & 1.00 & $1.04(0.70-1.55)$ & $1.64(1.12-2.41)$ & $1.98(1.36-2.90)$ & $1.30(1.11-1.56)$ & $1.88(1.42-2.48)$ \\
Model 2 & 1.00 & $1.01(0.66-1.52)$ & $1.62(1.10-2.42)$ & $1.80(1.21-2.68)$ & $1.21(1.02-1.49)$ & $1.81(1.35-2.42)$ \\
Model 3 & 1.00 & $1.06(0.70-1.60)$ & $1.72(1.16-2.54)$ & $1.91(1.29-2.83)$ & $1.31(1.09-1.61)$ & $1.76(1.32-2.33)$ \\
\hline
\end{tabular}

Model 1: age, sex, body mass index, daily alcohol intake, current smoking, comorbidities (hypertension, diabetes mellitus, dyslipidaemia, chronic kidney disease and atrial fibrillation), previous stroke, previous ischaemic heart disease, National Institutes Health Stroke Scale, ALP levels (quartiles range or 1-SD increase).

Model 2; the other blood laboratory findings were added to model 1.

Model 3: Calculated scores (CONUT, GNRI, NLR, PLR, and CAR) were added to model 1.

ALP, alkaline phosphatase; CONUT, Controlling Nutritional Status; GNRI, Geriatric Nutritional Risk Index; NLR, neutrophil-to-lymphocyte ratio; PLR, platelet-to-lymphocyte ratio; CAR, $\mathrm{C}$ reactive protein-to-albumin ratio

although those associations were not significant according to the analysis of model 2. Conversely, a $1-S D$ increase in ALP levels was independently associated with mRS scores of 3-6 at 3 months among patients with a premorbid $\mathrm{mRS}$ score of $0-1$ in each model (OR 1.32 95\% CI 1.10-1.59, $P<0.001$, model 1, OR 1.24, 95\% CI 1.01-1.53, $P=0.041$, model 2 and OR 1.34, 95\% CI 1.09-1.66, $P=0.002$, model 3).

\section{Discussion}

In the present study, by using multivariate linear discriminant or neural network analysis, ALP has been found to be a promising biological marker for improving the predictive ability for stroke outcomes when added to age, sex, and the NIHSS score. Additionally, after adjusting for baseline characteristics and other blood parameters, increased ALP levels were independently associated with poor stroke outcome.

A neural network analysis, a kind of machine learning, is an algorithm that works with a large number or possible predictor variables. Recently, this method has been applied in the medical field because it can predict disease severity or classification. In the present study, by using traditional or neural network analysis, ALP and nutritional status (CONUT score, albumin, or GNRI) have been found to be promising biological markers to predict functional stroke outcome. Remarkably, almost all AUC values used to predict poor outcomes with LLGMN were relatively higher than the AUC values obtained using a traditional analysis. Thus, the machine learning method may be superior to the traditional method. The CONUT score, an index calculated from the serum albumin concentration, total peripheral lymphocyte count, and total cholesterol concentration, is a screening tool for nutritional evaluation that has been validated in hospital populations ${ }^{5}$. We reported previously that malnutritional status using the CONUT score was independently associated with poor stroke outcomes among acute ischemic stroke $(n=230)^{4}$. Compared with that in our previous study, in the present study, the CONUT score has been confirmed to be a useful indicator for predicting stroke outcome in a large cohort. Only albumin might be a useful marker for predicting stroke outcome from our prediction models, although albumin is a component factor of the CONUT score. Indeed, several clinical studies have reported that reduced serum albumin levels in acute stroke patients were associated with poor outcomes ${ }^{23,24)}$. The GNRI, which was calculated from serum albumin levels and BMI, was also associated with poor stroke outcomes $^{25}$. In any case, assessments of nutritional status at admission are essential among acute ischemic stroke patients because malnourished stroke patients have a higher frequency of pneumonia, other infections, gastrointestinal bleeding, and bedsores than nourished patients ${ }^{26}$. Our findings provided further evidence that several nutritional indicators selected from prediction models were important factors for predicting stroke outcome.

Interestingly, besides nutritional status, serum ALP levels were ranked highly for improving the predictive ability for stroke outcome from both multivariate linear discriminant and neural network analyses. Therefore, we focused on the association between ALP levels, baseline characteristics, and stroke outcome in the present study. Serum ALP is usually used as a marker of liver disease or biliary obstructive disease in clinical settings. Recently, several studies have found that increased ALP levels were associated with cardiovascular events or mortality in the general 
population ${ }^{27-29)}$. Kunutsor et al. reported that the multivariate relative risk for cardiovascular events per 1-SD change in baseline ALP was 1.09 (95\% CI $1.02-1.16)$ in a meta-analysis ${ }^{30)}$. Additionally, studies regarding ALP as a prognostic stroke biological marker ${ }^{31-33)}$ had been accumulated. In the present study, we found that increased ALP levels at admission were independently associated with poor outcome at 3 months post-stroke onset after adjustments for other important parameters, including nutritional status related to stroke outcome.

Several possible mechanisms for the association between increased ALP levels and poor outcomes after stroke have been proposed. First, increased ALP levels have been implicated in the pathogenesis of vascular calcification ${ }^{34)}$. Vascular calcification is known to cause cardiovascular morbidity and mortality, and aortic calcification increases the relative risk of stroke ${ }^{35,36)}$. Second, serum ALP levels are also associated with inflammation or malnutrition ${ }^{33,37)}$. Indeed, in the present study, increased ALP levels were associated with CRP levels, D-dimer levels, and several nutritional statuses. However, we found that increased ALP levels were independently associated with poor stroke outcome after adjustment for those indicators. Hence, the association between ALP and poor stroke outcome is less likely to be merely dependent on malnutritional status and inflammation. Third, several basic studies have shown that ALP activity can be expressed in brain endothelial cells and neurons ${ }^{38,39)}$. Lee et al. found that increased ALP levels were associated with the presence of cerebral small vessel disease ${ }^{40)}$. These basic and clinical findings might indicate that ALP plays a role in the blood-brain barrier (BBB) breakdown, neuroinflammation, and vascular dysfunction in stroke patients ${ }^{41)}$. The mechanisms underlying the associations between increased ALP levels, neuroinflammation, BBB permeability, and vascular homeostasis might contribute to poor stroke outcomes ${ }^{41)}$.

Our study has several limitations. First, we excluded patients with intravenous thrombolysis or endovascular therapy because those therapies are strongly associated with stroke outcome. Additionally, not all subjects could be evaluated for blood laboratory parameters, which might lead to potential selection bias. Second, the AUC of ALP levels alone as a predictor of poor outcomes was not very high. Additionally, a 1-SD increase in ALP levels was not independently associated with secondary outcomes (mRS score of 2-6 at 3 months or mortality) after adjusting for other laboratory findings from blood samples (model 2). A larger validation cohort may be required to verify the usefulness of ALP levels as a predictor of various stroke outcomes. Third, we could not evaluate serum phosphate levels or 25-hydroxyvitamin D levels. We were also unable to evaluate bone disease affecting serum ALP levels. Interactions between ALP, phosphate, and vitamin D levels are associated with calcium and bone metabolism. Fourth, we did not evaluate detailed information on liver diseases that affect serum ALP levels. However, we could evaluate the daily alcohol intake and other liver enzymes. Multivariable analysis was conducted and adjusted for those influences. Serum ALP included several isoenzymes derived from the bone, liver, intestine, and so on. Further studies may be needed to elucidate the mechanism of association between increased specific ALP levels and poor stroke outcome.

To conclude, we determined prognostic factors such as ALP levels and nutritional status from prediction models of the multivariate linear discriminant and neural network analyses among acute ischemic stroke patients. These indicators were independently associated with functional outcome. Nutritional management is necessary for improving stroke outcome, and accurate nutritional intervention may prevent weight loss and enhance the muscle strength and quality of life of malnourished patients with stroke ${ }^{42)}$. Recently, a novel treatment that lowers ALP levels has been proposed to reduce the risk of cardiovascular events ${ }^{43)}$. Active nutritional intervention and ALPtargeted treatments will be expected for acute ischemic stroke patients.

\section{Sources of Funding}

This study was supported by research grant from the Japan Society for the Promotion of Science KAKENHI (Grant Numbers 20K16579).

\section{Disclosures}

Hirofumi Maruyama received grants from the Daiichi Sankyo Co., Ltd.; these grants are unrelated to the submitted work.

All other authors declare that they have no conflicts of interest.

\section{References}

1) Weimar C, König IR, Kraywinkel K, Ziegler A, Diener HC, Collaboration GSS. Age and national institutes of health stroke scale score within 6 hours after onset are accurate predictors of outcome after cerebral ischemia: Development and external validation of prognostic models. Stroke, 2004; 35: 158-162

2) König IR, Ziegler A, Bluhmki E, Hacke W, Bath PM, 
Sacco RL, et al. Predicting long-term outcome after acute ischemic stroke: A simple index works in patients from controlled clinical trials. Stroke, 2008; 39: 1821-1826

3) Collaboration GSS. Predicting outcome after acute ischemic stroke: An external validation of prognostic models. Neurology, 2004; 62: 581-585

4) Naito H, Nezu T, Hosomi N, Aoki S, Kinoshita N, Kuga $\mathrm{J}$, et al. Controlling nutritional status score for predicting 3 -mo functional outcome in acute ischemic stroke. Nutrition, 2018; 55-56: 1-6

5) Ignacio de Ulíbarri J, González-Madroño A, de Villar NG, González P, González B, Mancha A, et al. Conut: A tool for controlling nutritional status. First validation in a hospital population. Nutr Hosp, 2005; 20: 38-45

6) Zhang Y, Jiang L, Yang P. Comparison of lymphocyte count, neutrophil to lymphocyte ratio and platelet to lymphocyte ratio in predicting the severity and the clinical outcomes of acute cerebral infarction patients. Clin Lab, 2019; 65

7) Wang L, Song Q, Wang C, Wu S, Deng L, Li Y, et al. Neutrophil to lymphocyte ratio predicts poor outcomes after acute ischemic stroke: A cohort study and systematic review. J Neurol Sci, 2019; 406: 116445

8) Altintas O, Altintas MO, Tasal A, Kucukdagli OT, Asil T. The relationship of platelet-to-lymphocyte ratio with clinical outcome and final infarct core in acute ischemic stroke patients who have undergone endovascular therapy. Neurol Res, 2016; 38: 759-765

9) Mazaheri S, Reisi E, Poorolajal J, Ghiasian M. C-reactive protein levels and clinical outcomes in stroke patients: A prospective cohort study. Arch Iran Med, 2018; 21: 8-12

10) Matsuo R, Ago T, Hata J, Wakisaka Y, Kuroda J, Kuwashiro T, et al. Plasma c-reactive protein and clinical outcomes after acute ischemic stroke: A prospective observational study. PLoS One, 2016; 11: e0156790

11) Kubo S, Hosomi N, Hara N, Neshige $S$, Himeno $T$, Takeshima $S$, et al. Ischemic stroke mortality is more strongly associated with anemia on admission than with underweight status. J Stroke Cerebrovasc Dis, 2017; 26: 1369-1374

12) Yang XY, Gao S, Ding J, Chen Y, Zhou XS, Wang JE. Plasma d-dimer predicts short-term poor outcome after acute ischemic stroke. PLoS One, 2014; 9: e89756

13) Nezu T, Kitano T, Kubo S, Uemura J, Yamashita S, Iwanaga $T$, et al. Impact of d-dimer levels for short-term or long-term outcomes in cryptogenic stroke patients. J Neurol, 2018; 265: 628-636

14) Widhi Nugroho A, Arima $H$, Miyazawa I, Fujii T, Miyamatsu N, Sugimoto Y, et al. The association between glomerular filtration rate estimated on admission and acute stroke outcome: The shiga stroke registry. J Atheroscler Thromb, 2018; 25: 570-579

15) Shima K, Tsuji T, Kandori A, Yokoe M, Sakoda S. Measurement and evaluation of finger tapping movements using log-linearized gaussian mixture networks. Sensors (Basel), 2009; 9: 2187-2201

16) Tsuji T, Bu N, Fukuda O, Kaneko M. A recurrent log-linearized gaussian mixture network. IEEE Trans Neural Netw, 2003; 14: 304-316

17) Ichinose N, Hama S, Tsuji T, Soh Z, Hayashi H, Kiura Y, et al. Predicting ischemic stroke after carotid artery stent- ing based on proximal calcification and the jellyfish sign. J Neurosurg, 2018; 128: 1280-1288

18) Matsuo S, Imai E, Horio M, Yasuda Y, Tomita K, Nitta K, et al. Revised equations for estimated gfr from serum creatinine in japan. Am J Kidney Dis, 2009; 53: 982-992

19) Adams HP, Bendixen BH, Kappelle LJ, Biller J, Love BB, Gordon DL, et al. Classification of subtype of acute ischemic stroke. Definitions for use in a multicenter clinical trial. Toast. Trial of org 10172 in acute stroke treatment. Stroke, 1993; 24: 35-41

20) Bouillanne O, Morineau G, Dupont C, Coulombel I, Vincent JP, Nicolis I, et al. Geriatric nutritional risk index: A new index for evaluating at-risk elderly medical patients. Am J Clin Nutr, 2005; 82: 777-783

21) $\mathrm{Xu} \mathrm{XL}, \mathrm{Yu} \mathrm{HQ}, \mathrm{Hu}$ W, Song Q, Mao WM. A novel inflammation-based prognostic score, the c-reactive protein/albumin ratio predicts the prognosis of patients with operable esophageal squamous cell carcinoma. PLoS One, 2015; 10: e0138657

22) Suzuki T, Kudo A. Recent application of quantification ii in japanese medical research. Environ Health Perspect, 1979; 32: 131-141

23) Dziedzic T, Slowik A, Szczudlik A. Serum albumin level as a predictor of ischemic stroke outcome. Stroke, 2004; 35: e156-158

24) Babu MS, Kaul S, Dadheech S, Rajeshwar K, Jyothy A, Munshi A. Serum albumin levels in ischemic stroke and its subtypes: Correlation with clinical outcome. Nutrition, 2013; 29: 872-875

25) Kang MK, Kim TJ, Kim Y, Nam KW, Jeong HY, Kim $\mathrm{SK}$, et al. Geriatric nutritional risk index predicts poor outcomes in patients with acute ischemic stroke - automated undernutrition screen tool. PLoS One, 2020; 15: e0228738

26) Collaboration FT. Poor nutritional status on admission predicts poor outcomes after stroke: Observational data from the food trial. Stroke, 2003; 34: 1450-1456

27) Kunutsor SK, Bakker SJ, Kootstra-Ros JE, Gansevoort RT, Gregson J, Dullaart RP. Serum alkaline phosphatase and risk of incident cardiovascular disease: Interrelationship with high sensitivity c-reactive protein. PLoS One, 2015; 10: e0132822

28) Tonelli M, Curhan G, Pfeffer M, Sacks F, Thadhani R, Melamed ML, et al. Relation between alkaline phosphatase, serum phosphate, and all-cause or cardiovascular mortality. Circulation, 2009; 120: 1784-1792

29) Abramowitz M, Muntner P, Coco M, Southern W, Lotwin I, Hostetter TH, et al. Serum alkaline phosphatase and phosphate and risk of mortality and hospitalization. Clin J Am Soc Nephrol, 2010; 5: 1064-1071

30) Kunutsor SK, Apekey TA, Khan H. Liver enzymes and risk of cardiovascular disease in the general population: A meta-analysis of prospective cohort studies. Atherosclerosis, 2014; 236: 7-17

31) Kim J, Song TJ, Song D, Lee HS, Nam CM, Nam HS, et al. Serum alkaline phosphatase and phosphate in cerebral atherosclerosis and functional outcomes after cerebral infarction. Stroke, 2013; 44: 3547-3549

32) Zong L, Wang X, Li Z, Zhao X, Liu L, Li H, et al. Alkaline phosphatase and outcomes in patients with preserved renal function: Results from china national stroke registry. 
Stroke, 2018; 49: 1176-1182

33) Ryu WS, Lee SH, Kim CK, Kim BJ, Yoon BW. Increased serum alkaline phosphatase as a predictor of long-term mortality after stroke. Neurology, 2010; 75: 1995-2002

34) Johnson RC, Leopold JA, Loscalzo J. Vascular calcification: Pathobiological mechanisms and clinical implications. Circ Res, 2006; 99: 1044-1059

35) Adler Y, Fink N, Spector D, Wiser I, Sagie A. Mitral annulus calcification--a window to diffuse atherosclerosis of the vascular system. Atherosclerosis, 2001; 155: 1-8

36) Hollander M, Hak AE, Koudstaal PJ, Bots ML, Grobbee DE, Hofman A, et al. Comparison between measures of atherosclerosis and risk of stroke: The rotterdam study. Stroke, 2003; 34: 2367-2372

37) Wannamethee SG, Sattar N, Papcosta O, Lennon L, Whincup PH. Alkaline phosphatase, serum phosphate, and incident cardiovascular disease and total mortality in older men. Arterioscler Thromb Vasc Biol, 2013; 33: 1070-1076

38) Ermonval M, Baudry A, Baychelier F, Pradines E, Pietri $\mathrm{M}$, Oda $\mathrm{K}$, et al. The cellular prion protein interacts with the tissue non-specific alkaline phosphatase in membrane microdomains of bioaminergic neuronal cells. PLoS One, 2009; 4: e6497

39) Brun-Heath I, Ermonval M, Chabrol E, Xiao J, Palkovits M, Lyck R, et al. Differential expression of the bone and the liver tissue non-specific alkaline phosphatase isoforms in brain tissues. Cell Tissue Res, 2011; 343: 521-536

40) Lee HB, Kim J, Kim SH, Kim S, Kim OJ, Oh SH. Association between serum alkaline phosphatase level and cerebral small vessel disease. PLoS One, 2015; 10: e0143355

41) Brichacek AL, Brown CM. Alkaline phosphatase: A potential biomarker for stroke and implications for treatment. Metab Brain Dis, 2019; 34: 3-19

42) Ha L, Hauge T, Spenning AB, Iversen PO. Individual, nutritional support prevents undernutrition, increases muscle strength and improves qol among elderly at nutritional risk hospitalized for acute stroke: A randomized, controlled trial. Clin Nutr, 2010; 29: 567-573

43) Filippakopoulos P, Knapp S. The bromodomain interaction module. FEBS Lett, 2012; 586: 2692-2704 
Supplement Table 2. Correlation of alkaline phosphatase to laboratory findings

\begin{tabular}{lcc}
\hline & \multicolumn{2}{c}{ ALP $(n=1,484)$} \\
Laboratory findings & $\rho$ & $P$ \\
\hline Haemoglobin $(\mathrm{g} / \mathrm{dl})$ & 0.025 & 0.34 \\
White blood cell $(/ \mu \mathrm{l})(n=1,483)$ & 0.041 & 0.11 \\
Lymphocyte count $(/ \mu \mathrm{l})(n=1,481)$ & -0.024 & 0.35 \\
Neutrophil count $(/ \mu \mathrm{l})(n=1,481)$ & 0.051 & 0.049 \\
Platelet $\left(\times 10^{4} / \mu \mathrm{l}\right)(n=1,482)$ & 0.053 & 0.042 \\
Albumin $(\mathrm{g} / \mathrm{dl})(n=1,475)$ & -0.065 & 0.012 \\
Total cholesterol $(\mathrm{mg} / \mathrm{dl})(n=1,474)$ & -0.028 & 0.28 \\
LDL cholesterol $(\mathrm{mg} / \mathrm{dl})(n=1,455)$ & 0.006 & 0.83 \\
HDL cholesterol $(\mathrm{mg} / \mathrm{dl})(n=1,453)$ & -0.098 & $<0.001$ \\
TG $(\mathrm{mg} / \mathrm{dl})(n=1,466)$ & 0.020 & 0.45 \\
Cholinesterase $(\mathrm{U} / \mathrm{I})(n=1,424)$ & -0.041 & 0.12 \\
AST $(\mathrm{U} / \mathrm{I})$ & 0.103 & $<0.001$ \\
ALT $(\mathrm{U} / \mathrm{I})$ & 0.078 & $<0.001$ \\
LDH $(\mathrm{U} / \mathrm{I})(n=1,478)$ & 0.104 & $<0.001$ \\
$\gamma$-GTP $(\mathrm{U} / \mathrm{I})(n=1,472)$ & 0.137 & $<0.001$ \\
BUN $(\mathrm{mg} / \mathrm{dl})$ & -0.018 & 0.50 \\
Cr $(\mathrm{mg} / \mathrm{dl})$ & -0.074 & 0.005 \\
eGFR $\left(\mathrm{L} / \mathrm{min} / 1.73 \mathrm{~m}{ }^{2}\right)$ & 0.041 & 0.12 \\
HbA1c $(\%)(n=1,450)$ & 0.002 & 0.94 \\
CRP $(\mathrm{mg} / \mathrm{dl})(n=1,477)$ & 0.161 & $<0.001$ \\
D-dimer $(\mu \mathrm{mg} / \mathrm{mL})(n=1,435)$ & 0.106 & $<0.001$ \\
\hline
\end{tabular}

LDL, low-density lipoprotein; HDL, high-density lipoprotein; TG, triglyceride; AST, aspartate aminotransferase; ALT, alanine aminotransferase; LDH, lactate dehydrogenase; $\gamma$ -GTP, $\gamma$-glutamyl transpeptidase; BUN, blood urea nitrogen; Cr, creatinine; HbAlc, haemoglobin A1c; CRP, C-reactive protein.

Supplement Table 3. Laboratory findings associated with 3-month functional outcome

\begin{tabular}{|c|c|c|c|}
\hline & mRS $0-2(n=960)$ & mRS 3-6 $(n=524)$ & $p$ \\
\hline Haemoglobin $(\mathrm{g} / \mathrm{dl})$ & $13.7 \pm 2.1$ & $13.0 \pm 2.3$ & $<0.001$ \\
\hline White blood cell $(/ \mu \mathrm{l})(n=1,483)$ & $7016.7 \pm 2686.2$ & $7943.8 \pm 3641.0$ & $<0.001$ \\
\hline Lymphocyte count $(/ \mu \mathrm{l})(n=1,481)$ & $1720.8 \pm 823.8$ & $1415.9 \pm 719.5$ & $<0.001$ \\
\hline Neutrophil count $(/ \mu \mathrm{l})(n=1,481)$ & $4692.5 \pm 2277.4$ & $5981.8 \pm 3525.6$ & $<0.001$ \\
\hline Platelet $\left(\times 10^{4} / \mu \mathrm{l}\right)(n=1,482)$ & $20.8 \pm 7.6$ & $19.3 \pm 9.4$ & 0.001 \\
\hline Albumin (g/dl) $(n=1,475)$ & $4.1 \pm 0.5$ & $3.8 \pm 0.6$ & $<0.001$ \\
\hline Total cholesterol $(\mathrm{mg} / \mathrm{dl})(n=1,474)$ & $194.5 \pm 42.9$ & $188.9 \pm 47.3$ & 0.020 \\
\hline LDL cholesterol $(\mathrm{mg} / \mathrm{dl})(n=1,455)$ & $116.3 \pm 36.9$ & $114.6 \pm 38.3$ & 0.41 \\
\hline HDL cholesterol $(\mathrm{mg} / \mathrm{dl})(n=1,453)$ & $52.0 \pm 15.4$ & $51.0 \pm 14.6$ & 0.22 \\
\hline TG $(\mathrm{mg} / \mathrm{dl})(n=1,466)$ & $130.8 \pm 92.7$ & $108.4 \pm 77.3$ & $<0.001$ \\
\hline Cholinesterase (U/I) $(n=1,424)$ & $296.2 \pm 79.6$ & $260.4 \pm 86.7$ & 0.002 \\
\hline AST (U/I) & $25.1 \pm 12.9$ & $31.3 \pm 28.0$ & $<0.001$ \\
\hline $\operatorname{ALT}(\mathrm{U} / \mathrm{I})$ & $22.3 \pm 17.6$ & $24.2 \pm 55.5$ & 0.34 \\
\hline $\mathrm{LDH}(\mathrm{U} / \mathrm{I})(n=1,478)$ & $210.9 \pm 57.2$ & $261.8 \pm 134.2$ & $<0.001$ \\
\hline$\gamma-\operatorname{GTP}(\mathrm{U} / \mathrm{I})(n=1,472)$ & $43.7 \pm 58.3$ & $45.8 \pm 84.9$ & 0.57 \\
\hline BUN (mg/dl) & $17.9 \pm 10.2$ & $20.8 \pm 12.8$ & $<0.001$ \\
\hline $\mathrm{Cr}(\mathrm{mg} / \mathrm{dl})$ & $1.14 \pm 1.61$ & $1.07 \pm 1.12$ & 0.41 \\
\hline eGFR (L/min/1.73 $\left.\mathrm{m}^{2}\right)$ & $67.0 \pm 24.6$ & $63.2 \pm 27.5$ & 0.007 \\
\hline $\operatorname{HbA1c}(\%)(n=1,450)$ & $6.1 \pm 1.2$ & $6.1 \pm 1.1$ & 0.65 \\
\hline $\mathrm{CRP}(\mathrm{mg} / \mathrm{dl})(n=1,477)$ & $0.17(0.1-0.4)$ & $0.3(0.1-1.2)$ & $<0.001$ \\
\hline D-dimer $(\mu \mathrm{g} / \mathrm{mL})(n=1,435)$ & $0.9(0.6-1.4)$ & $1.7(0.9-3.8)$ & $<0.001$ \\
\hline
\end{tabular}

